\title{
Optimism and Pessimism in Decision Making Based on Intuitionistic Fuzzy Sets
}

\author{
Ting-Yu Chen ${ }^{1}$ Che-Wei Tsui ${ }^{2}$ \\ ${ }^{1}$ Department of Business Administration, Chang Gung University \\ ${ }^{2}$ Graduate Institute of Industrial Engineering and Engineering Management, National \\ Tsing Hua University
}

\begin{abstract}
This paper presents a method of relating optimism and pessimism to multiple criteria decision analysis based on intuitionistic fuzzy sets. We develop the concepts of optimistic and pessimistic point operators to measure optimism and pessimism, respectively. Furthermore, we provide an approach to effectively capture the influences of optimism and pessimism on multiple criteria decision making. The suitability function is assessed according to the influence upon overall judgments or upon individual outcomes. Finally, we establish two algorithms to solve a multicriteria decision making problem.
\end{abstract}

Keywords: Optimism, pessimism, multiple criteria decision analysis, intuitionistic fuzzy set, point operator

\section{Introduction}

Intuitionistic fuzzy set (IFS for short), introduced by Atanassov (1986, 1999), is characterized by three functions expressing the degree of belongingness, the degree of nonbelongingness, and the degree of hesitation. IFSs have been found to be highly useful to deal with uncertainty and vagueness (Xu and Yager, 2008) and have become a popular topic of investigation in the fuzzy set community (Dubois et al., 2005). Although a considerable number of studies have been made on intuitionistic fuzzy multiple criteria deci- sion making, little attention has been given to the issue of optimism and pessimism. Optimism and pessimism are fundamental inner psychological characteristics that both determine and reflect how a person responds to his or her perceived environment. The evidence in psychology suggests that one main channel through which dispositional optimism works is through developing coping habits or behavior that is more likely to lead to desired outcomes (Friedman et al., 1995; Puri and Robinson, 2007).

In view of multi-criteria decision making problems, optimism and pessimism can reflect individual differences of different decision makers. In addition, they tend to be both consistent and enduring. It follows that optimism and pessimism consistently influence how the decision maker responds to the decision environment. Therefore, the identification of their influence on the decision making process is highly valuable and useful in multiple criteria decision analysis. In this research, we will develop optimistic and pessimistic estimations based on IFSs to multi-criteria decision analysis, where optimism and pessimism are measured by using optimistic and pessimistic point operators, respectively. In addition, several important properties will be examined and discussed. For decision aiding, we provide an approach relating optimism and pessimism to multi-criteria decision analysis under the intuitionistic fuzzy decision environment.

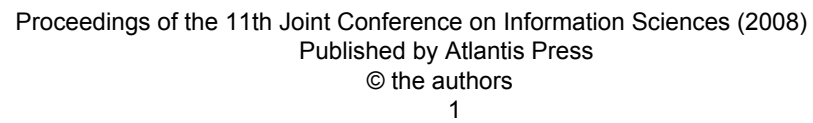




\section{Preliminaries}

The concept of IFSs is a generalization ordinary fuzzy sets. We briefly review some relevant definitions, relations, and operations of IFSs.

Definition 2.1. Let $X$ be an ordinary finite non-empty set. An IFS $A$ in $X$ is an expression given by:

$$
A=\left\{\left\langle x, \mu_{A}(x), v_{A}(x)\right\rangle \mid x \in X\right\},
$$

where $\mu_{A}(x): X \rightarrow[0,1], \quad v_{A}(x): X \rightarrow[0,1]$ with $0 \leq \mu_{A}(x)+v_{A}(x) \leq 1$. The numbers $\mu_{A}(x)$ and $v_{A}(x)$ denote the membership degree and the non-membership degree of the element $x$ in $A$, respectively.

Definition 2.2. For each IFS $A$ in $X$, the value of

$$
\pi_{A}(x)=1-\mu_{A}(x)-v_{A}(x)
$$

represents the the intuitionistic index.

Definition 2.3. For every IFS $A$ and for every $\alpha, \beta \in[0,1]$ :

$$
\begin{aligned}
& J_{\alpha, \beta}(A)=\left\{\left\langle x, \mu_{A}(x)+\alpha \cdot \pi_{A}(x), \beta \cdot v_{A}(x)\right\rangle \mid x \in X\right\} \\
& J_{\alpha, \beta}^{*}(A)=\left\{\left\langle x, \mu_{A}(x)+\alpha \cdot\left(1-\mu_{A}(x)-\beta \cdot v_{A}(x)\right), \beta \cdot v_{A}(x)\right\rangle \mid x \in X\right\} \\
& H_{\alpha, \beta}(A)=\left\{\left\langle x, \alpha \cdot \mu_{A}(x), v_{A}(x)+\beta \cdot \pi_{A}(x)\right\rangle \mid x \in X\right\} \\
& H_{\alpha, \beta}^{*}(A)=\left\{\left\langle x, \alpha \cdot \mu_{A}(x), v_{A}(x)+\beta \cdot\left(1-\alpha \cdot \mu_{A}(x)-v_{A}(x)\right)\right\rangle \mid x \in X\right\} \\
& P_{\alpha, \beta}(A)=\left\{\left\langle x, \max \left(\mu_{A}(x), \alpha\right), \min \left(v_{A}(x), \beta\right)\right\rangle \mid x \in X\right\} \\
& Q_{\alpha, \beta}(A)=\left\{\left\langle x, \min \left(\mu_{A}(x), \alpha\right), \max \left(v_{A}(x), \beta\right)\right\rangle \mid x \in X\right\}
\end{aligned}
$$

\section{Optimistic and Pessimistic Estima- tions on IFSs}

This paper relates optimism and pessimism to multi-criteria decision making behavior and establishes appropriate assessment tools for measuring them in decision analysis. Optimists construe their lives and future states of the world positively, whereas pessimists construe their lives and future states of the world negatively. In addition, optimists expect greater overall utility or favorable outcomes, but pessimists expect less overall utility or unfavorable outcomes. The above rationale coincides with several Atanassov's operators, including $J_{\alpha, \beta}, J_{\alpha, \beta}^{*}$, $H_{\alpha, \beta}, H_{\alpha, \beta}^{*}, P_{\alpha, \beta}$ and $Q_{\alpha, \beta}$. By extension we will develop new point operators denoted by $J_{\alpha_{x}, \beta_{x}}, J_{\alpha_{x}, \beta_{x}}^{*}, H_{\alpha_{x}, \beta_{x}}, H_{\alpha_{x}, \beta_{x}}^{*}, P_{\alpha_{x}, \beta_{x}}$ and $Q_{\alpha_{x}, \beta_{x}}$ for each point $x \in X$ and $\alpha_{x}, \beta_{x} \in[0,1]$. These estimations on IFSs are called as optimistic or pessimistic point operators.

Definition 3.1. For each $x \in X$, taking $\alpha_{x}, \beta_{x} \in[0,1]$ we define optimistic point operators $J_{\alpha_{s} \beta_{x}}, J_{\alpha_{s} \beta_{x}}^{*}, P_{\alpha_{\alpha} \beta_{x}}: \operatorname{IFS}(X) \rightarrow \operatorname{IFS}(X)$ as follows, for $A \in \operatorname{IFS}(X)$ :

$J_{\alpha_{x}, \beta_{x}}(A)=\left\{\left\langle x, \mu_{A}(x)+\alpha_{x} \cdot \pi_{A}(x), \beta_{x} \cdot v_{A}(x)\right\rangle \mid x \in X\right\}$

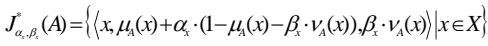

$P_{a_{x}, \beta_{x}}(A)=\left\{\left\langle x, \max \left(\mu_{A}(x), \alpha_{x}\right), \min \left(v_{A}(x), \beta_{x}\right)\right\rangle \mid x \in X\right\}$ for ${ }_{\alpha_{x}+\beta_{x} \leq 1}$

Definition 3.2. For each $x \in X$, taking $\alpha_{x}, \beta_{x} \in[0,1]$ we define pessimistic point operators $H_{\alpha_{x} \beta_{x}}, H_{\alpha_{x}, \beta_{x}}^{*}, Q_{\alpha_{x} \beta_{x}}: \operatorname{IFS}(X) \rightarrow \operatorname{IFS}(X)$ as follows, for $A \in \operatorname{IFS}(X)$ :

$$
\begin{aligned}
& H_{\alpha_{x}, \beta_{x}}(A)=\left\{\left\langle x, \alpha_{x} \cdot \mu_{A}(x), v_{A}(x)+\beta_{x} \cdot \pi_{A}(x)\right\rangle \mid x \in X\right\} \\
& H_{\alpha_{x}, \beta_{x}}^{\cdot}(A)=\left\{\left\langle x, \alpha_{x} \cdot \mu_{A}(x), v_{A}(x)+\beta_{x} \cdot\left(1-\alpha_{x} \cdot \mu_{A}(x)-v_{A}(x)\right)\right\rangle \mid x \in X\right\} \\
& Q_{\alpha_{x}, \beta_{x}}(A)=\left\{\left\langle x, \min \left(\mu_{A}(x), \alpha_{x}\right), \max \left(v_{A}(x), \beta_{x}\right)\right\rangle \mid x \in X\right\}
\end{aligned}
$$

Definition 3.3 Let $\kappa, \kappa_{1}, \kappa_{2}$ be real numbers and let $a_{1}, a_{2}, \cdots, a_{n} \in[0,1]$. For $n$ IFSs $A_{1}, A_{2}, \cdots, A_{n}$, we define the averaging operations based on generalized means:

$$
\begin{aligned}
& A_{1} \underset{\kappa_{1}, \kappa_{2}}{\otimes} A_{2}=\bigotimes_{i=1}^{2} A_{i} \\
& \text { and } \\
& \bigotimes_{i=1}^{n} A_{i}=\left\{\left\langlex, f\left(\kappa_{1}, \mu_{A_{1}}(x), \mu_{A_{2}}(x), \cdots, \mu_{A_{n}}(x)\right),\right.\right. \\
& \left.f\left(\kappa_{2}, v_{A_{1}}(x), v_{A_{2}}(x), \cdots, v_{A_{n}}(x)\right)|x \in X\rangle\right\}
\end{aligned}
$$


where

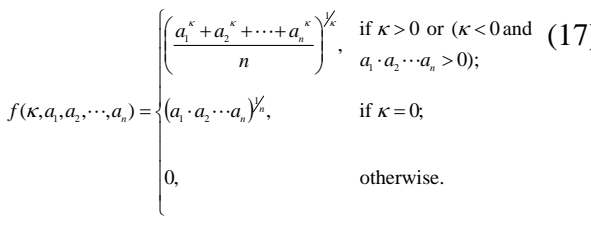

\section{Application in Multi-Criteria Deci- sion Making}

This section presents an approach for handling optimism and pessimism in multi-criteria decision making problems under the intuitionistic fuzzy environment. By similar definitions of $\mathrm{Li}$ (2005) and Lin et al. (2007), the evaluations of each alternative with respect to each criterion on a fuzzy concept "excellence" are given using IFSs. Suppose that there exists a non-inferior alternative set $X=\left\{x_{1}, x_{2}, \ldots, x_{m}\right\}$. Each alternative is assessed on $n$ criteria, denoted by $A=\left\{A_{1}, A_{2}, \ldots, A_{n}\right\}$. Assume that $\mu_{i j}$ and $v_{i j}$ are the degree of membership and the degree of non-membership of the alternative $x_{j} \in X$ with respect to the criterion $A_{i} \in A$ to the fuzzy concept "excellence", respectively, where $0 \leq \mu_{i j} \leq 1$, $0 \leq v_{i j} \leq 1$ and $0 \leq \mu_{i j}+v_{i j} \leq 1$. Denote that $X_{i j}=\left\{\left\langle x_{j}, \mu_{i j}, v_{i j}\right\rangle\right\}$. The intuitionistic index of the alternative $x_{j}$ in the set $X_{i j}$ is defined by c.

\subsection{An approach utilizing optimistic and pessimistic point operators}

Assume that the decision maker consider evaluative criteria all equally important for simplicity. The suitability function to determine the degrees to which the alternative $x_{j}$ satisfies and does not satisfy the decision maker's requirement can be measured as follows:

$$
\bigotimes_{i=1}^{n} A_{i}=\left\{\left\langle x_{j}, f\left(\kappa_{1}, \mu_{i j}, \mu_{2 j}, \cdots, \mu_{n j}\right), f\left(\kappa_{2}, v_{1 j}, v_{2 j}, \cdots, v_{n j}\right)\right\rangle x_{j} \in X\right\}
$$

If an optimistic decision maker construe overall judgment positively and expect favorable synthetic evaluation, the suitability function to determine the degrees to which the alternative $x_{j}$ satisfies and does not satisfy the decision maker's requirement becomes $J_{\alpha_{x_{t}, \beta_{i j}}}\left({ }_{i=1}^{\otimes} A_{i}\right), J_{\alpha_{x_{j}}, \beta_{z_{i}}}^{*}\left(\otimes_{i=1}^{n} A_{i}\right)$ or $P_{\alpha_{x_{j}}, \beta_{x_{j}}}\left(\stackrel{n}{i=1}_{i}^{n} A_{i}\right)$. If an optimistic decision maker reconstructs the decision matrix with more desirable outcomes, the suitability function will be $\bigotimes_{i=1}^{n} J_{\alpha_{x_{j}}, \beta_{x_{j}}}\left(A_{i}\right)$, C or $\bigotimes_{i=1}^{n} P_{\alpha_{x_{j}}, \beta_{x_{j}}}\left(A_{i}\right)$.

On the contrary, if a pessimistic decision maker reflects negative attitude on overall judgments, the suitability function must adjust to $H_{\alpha_{x_{j}}, \beta_{x_{j}}}\left(\otimes_{i=1}^{n} A_{i}\right), H_{\alpha_{x_{j}}, \beta_{x_{j}}}^{*}\left(\otimes_{i=1}^{n} A_{i}\right)$ or $Q_{\alpha_{x_{j}}, \beta_{x_{j}}}\left(\otimes_{i=1}^{n} A_{i}\right)$. If a pessimistic decision maker modifies the decision matrix with more adverse outcomes, the corresponding suitability function is $\otimes_{i=1}^{n} H_{\alpha_{x_{j}}, \beta_{k_{j}}}\left(A_{i}\right)$, $\bigotimes_{i=1}^{n} H_{\alpha_{x_{j}}, \beta_{x_{j}}}^{*}\left(A_{i}\right)$ or $\otimes_{i=1}^{n} Q_{\alpha_{x_{j}}, \beta_{x_{j}}}\left(A_{i}\right)$. Then, measure the degree of suitability to which the alternative $x_{j}$ satisfies the decision maker's requirement on the basis of the score function.

Definition 4.1. Let $X=\left\{x_{1}, x_{2}, \ldots, x_{m}\right\}$ be the set of the considered alternatives, and let $A=\left\{A_{1}, A_{2}, \ldots, A_{n}\right\}$ be the set of the criteria used for evaluating the alternatives. Let $\alpha_{x_{i}}, \beta_{x_{i}} \in[0,1]$ for all $x_{j} \in X$. Assume that individual differences in virtue of optimism or pessimism influence overall judgments. The suitability function to determine the degrees to which the alternative $x_{j}$ satisfies and does not satisfy the decision maker's requirement can be measured as follows:

(i) For the optimistic condition: 


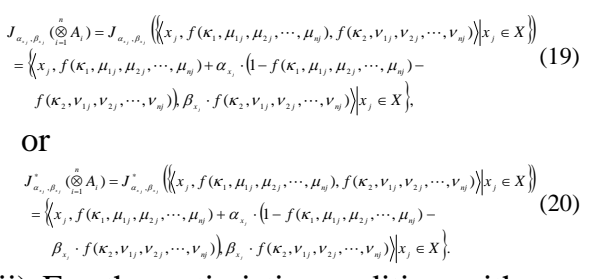

(ii) For the optimistic condition with restrictions:

$$
\begin{aligned}
& P_{\alpha_{1}, B_{x_{2}}}(\stackrel{n}{\otimes} A)
\end{aligned}
$$

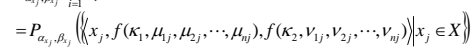

$$
\begin{aligned}
& =\left\{x_{j}, \max \left(f\left(\kappa_{1}, \mu_{1 j}, \mu_{2 j}, \cdots, \mu_{r_{j}}\right), \alpha_{x_{j}}\right),\right. \\
& \left.\left.\min \left(f\left(\kappa_{2}, v_{1 j}, v_{2 j}, \cdots, v_{r_{j}}\right), \beta_{x_{j}}\right)\right) \mid x_{j} \in X\right\} \text { for } \alpha_{x_{j}}+\beta_{x_{j}} \leq 1 \text {. }
\end{aligned}
$$

(iii) For the pessimistic condition:

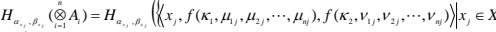

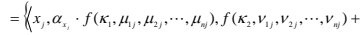

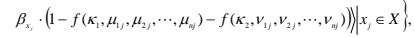

$$
\begin{aligned}
& \text { or }
\end{aligned}
$$

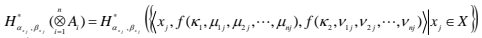

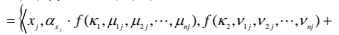

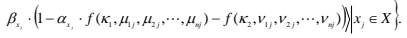

(iv) For the pessimistic condition with restrictions:

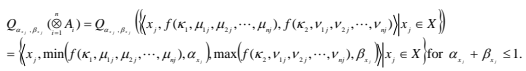

Definition 4.2. Let $X=\left\{x_{1}, x_{2}, \ldots, x_{m}\right\}$ be the set of the considered alternatives, and let $A=\left\{A_{1}, A_{2}, \ldots, A_{n}\right\}$ be the set of the criteria used for evaluating the alternatives. Let $\alpha_{x_{1}}, \beta_{x_{i}} \in[0,1]$ for all $x_{j} \in X$. Assume that individual differences in virtue of optimism or pessimism influence outcomes in the decision matrix. The suitability function to determine the degrees to which the alternative $x_{j}$ satisfies and does not satisfy the decision maker's requirement can be measured as follows:

(i) For the optimistic condition:

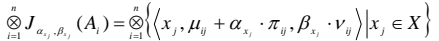

$$
\begin{aligned}
& =\left\{\left(x_{j}, f\left(\kappa_{1}, \mu_{1 j}+\alpha_{x_{j}} \cdot \pi_{1 j}, \mu_{2 j}+\alpha_{x_{j}} \cdot \pi_{2 j}, \cdots, \mu_{n j}+\alpha_{x_{j}} \cdot \pi_{n j}\right),\right.\right. \\
& \left.f\left(\kappa_{2}, \beta_{x_{j}} \cdot v_{1 j}, \beta_{x_{j}} \cdot v_{2 j}, \cdots, \beta_{x_{j}} \cdot v_{\mathrm{pj}}\right) \mid x_{j} \in X\right\}, \\
& \text { or }
\end{aligned}
$$

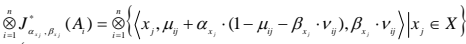

$$
\begin{aligned}
& =\left\{x_{i j}, f\left(\kappa_{1}, \mu_{1 j}+\alpha_{x_{i}} \cdot\left(1-\mu_{1 j}-\beta_{x j} \cdot v_{1 j}\right), \mu_{2 j}+\alpha_{x_{x}} \cdot\left(1-\mu_{2 j}-\beta_{x_{i}} \cdot v_{2 j}\right), \cdots,(26)\right.\right. \\
& \left.\mu_{i j}+\alpha_{x_{j}} \cdot\left(1-\mu_{i j}-\beta_{x^{\prime}} \cdot v_{i j}\right), f\left(\kappa_{2}, \beta_{x_{i}} \cdot v_{i j}, \beta_{x_{j}} \cdot v_{2, j}, \cdots, \beta_{x_{j}} \cdot v_{m i}\right) \mid x_{j} \in X\right\} .
\end{aligned}
$$

(ii) For the optimistic condition with restrictions:

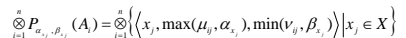

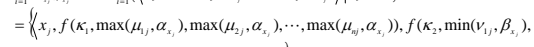

(iii) For the pessimistic condition:

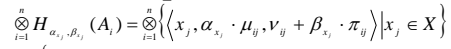

$$
\begin{aligned}
& =\left\{\left\langlex_{j}, f\left(\kappa_{1}, \alpha_{x_{1}} \cdot \mu_{1}, \alpha_{x_{j}} \cdot \mu_{2,}, \cdots, \alpha_{x_{j}} \cdot \mu_{n j}\right),\right.\right. \\
& \left.f\left(\kappa_{2}, v_{1 j}+\beta_{x_{j}} \cdot \pi_{1 j}, v_{2 j}+\beta_{x_{j}} \cdot \pi_{2 j}, \cdots, v_{n j}+\beta_{x_{j}} \cdot \pi_{n j}\right) \mid x_{j} \in X\right\}, \\
& \text { or }
\end{aligned}
$$

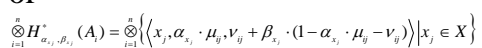

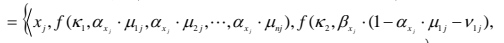

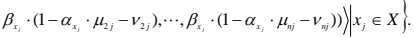

(iv) For the pessimistic condition with restrictions:

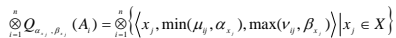

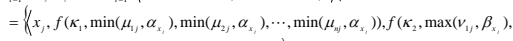

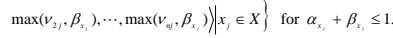

Based on the simple additive model, the steps for solving a multi-criteria decision making problem can be summed up as follows.

Algorithm (I): for the effect of optimism or pessimism upon overall judgments.

Step 1: Establish evaluation criteria $A_{i}$ 's which have been judged relevant in a given decision situation by the decision maker. Denote $A=\left\{A_{1}, A_{2}, \ldots, A_{n}\right\}$.

Step 2: Develop feasible alternatives $x_{j}^{\prime}$ s for achieving the goals or attaining the decision maker's needs and desires. Denote $X=\left\{x_{1}, x_{2}, \ldots, x_{m}\right\}$. Set $\alpha_{x_{j}}, \beta_{x_{j}}$ $\in[0,1]$ for all $x_{j} \in X$ according to the decision maker's preference information on alternatives.

Step 3: Evaluate alternatives in terms of criteria on the fuzzy concept "excellence", where the values of criterion functions are expressed by IFSs. Denote $X_{i j}=\left\{\left\langle x_{j}, \mu_{i j}, v_{i j}\right)\right\}$.

Step 4: Acquire the synthetic evaluation, unconsidering optimism or pessimism, of the alternative $x_{j}$ by employing the averaging operation in (18).

Step 5: Determine the suitability function of the alternative $x_{j}$ by employing the optimistic and pessimistic point operators. Use (19) or (20) for the optimistic 
condition, (21) for the optimistic condition with restrictions, (22) or (23) for the pessimistic condition, and (24) for the pessimistic condition with restrictions.

Step 6: Measure the degree of suitability for each alternative by means of the score function.

Step 7: Rank the preference order of all alternatives according to the degree of suitability. If there exists $j_{o} \in\{1,2, \cdots, m\}$ such that the score function is largest, then the alternative $x_{j_{o}}$ is the best choice.

Algorithm (II): for the effect of optimism or pessimism upon individual outcomes.

Steps 1-3: See Steps 1-3 of Algorithm (I). Step 4: Construct the intuitionistic fuzzy decision matrix that is composed of all $X_{i j}$ 's.

Step 5: Utilize the optimistic point operators, (9), (10), or (11), and pessimistic point operators, (12), (13), or (14), to reconstruct the intuitionistic fuzzy decision matrix.

Step 6: Determine the suitability function of the alternative $x_{j}$ by employing the averaging operation. Use (25) or (26) for the optimistic condition, (27) for the optimistic condition with restrictions, (28) or (29) for the pessimistic condition, and (30) for the pessimistic condition with restrictions.

Steps 7-8: See Steps 6-7 of Algorithm (I).

\subsection{Illustrative example}

Assume that the degrees $\mu_{i j}$ of membership and the degrees $v_{i j}$ of nonmembership for alternative $x_{j} \in X$ with respect to criterion $A_{i} \in A$ to the fuzzy concept “excellence” are give below:

$$
\begin{aligned}
& \text { A } \quad A_{2} \quad A \\
& x_{1}[(0.39,0.19) \quad(0.83,0.12) \quad(0.27,0.25)] \\
& \begin{array}{llll}
x_{2} & (0.27,0.55) & (0.73,0.17) & (0.54,0.19)
\end{array} \\
& \begin{array}{lllll}
\left(\left(\mu_{i j}, v_{i j}\right)\right)_{5 \times 3}=x_{3} & (0.34,0.51) & (0.68,0.01) & (0.55,0.27)
\end{array} \\
& x_{4}(0.63,0.16) \quad(0.48,0.20) \quad(0.30,0.41) \\
& \left.x_{5}(0.55,0.23) \quad(0.54,0.25) \quad(0.38,0.56)\right]
\end{aligned}
$$

We measure the degree of suitability of each alternative by employing all of optimistic and pessimistic point operators. Different settings of parameters are adopted, including (i) $\alpha_{x_{j}}=0.4, \beta_{x_{j}}=0.3$; (ii)

$\alpha_{x_{j}}=0.6, \beta_{x_{j}}=0.4$; (iii) $\alpha_{x_{j}}=0.2, \beta_{x_{j}}=0.7$, and (iv) $\alpha_{x_{j}}=\mu_{i j} /\left(\mu_{i j}+v_{i j}\right), \beta_{x_{j}}=v_{i j} /\left(\mu_{i j}+v_{i j}\right)$

\begin{tabular}{|c|c|c|c|c|}
\hline \multirow{3}{*}{$\begin{array}{l}\text { Suitability } \\
\text { function }\end{array}$} & \multicolumn{2}{|c|}{ Fixed point operator } & \multicolumn{2}{|c|}{ Variant point operator } \\
\hline & $\alpha_{x_{j}}=0.4$ & $\alpha_{x_{j}}=0.6$ & $\alpha_{x_{j}}=0.2$ & $\alpha_{x_{i}}=\mu_{i j} /\left(\mu_{i j}+v_{i j}\right)$ \\
\hline & $\beta_{x_{i}}=0.3$ & $\beta_{x_{j}}=0.4$ & $\beta_{x_{j}}=0.7$ & $\beta_{x_{i}}=v_{i} /\left(\mu_{i}+v_{i j}\right)$ \\
\hline \multirow{7}{*}{ 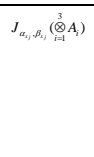 } & $x_{1}: 0.5673$ & $x_{1}: 0.6120$ & $x_{1}: 0.4293$ & $x_{1}: 0.6758$ \\
\hline & $x_{2}: 0.4957$ & $x_{2}: 0.5020$ & $x_{2}: 0.3377$ & $x_{2}: 0.5159$ \\
\hline & $x_{3}: 0.5297$ & $x_{3}: 0.5460$ & x: 0.3817 & $x_{3}: 0.5771$ \\
\hline & $x_{4}: 0.5023$ & $\begin{array}{l}x: 0.5313 \\
x=0.0493\end{array}$ & $\mathrm{x}_{4}: 0.3450$ & $x_{4}: 0.5561$ \\
\hline & $x_{5}: 0.4513$ & $x_{5}: 0.4493$ & $x_{5}: 0.2800$ & $x_{5}: 0.4420$ \\
\hline & $x_{1} \succ x_{3} \succ x_{4} \succ$ & $x_{1} \succ x_{3} \succ x_{4} \succ$ & $x_{1}>x_{3}>x_{4} \succ$ & $x_{1} \succ x_{3} \succ x_{4} \succ$ \\
\hline & $x_{2} \succ x_{5}$ & $x_{2} \succ x_{5}$ & $x_{2}>x_{5}$ & $x_{2}>x_{5}$ \\
\hline \multirow{7}{*}{$J_{\alpha_{\alpha_{i},}, B_{i}}^{*}\left(\bigotimes_{i=1}^{3} A_{i}\right)$} & $x_{1}: 0.6196$ & $x_{1}: 0.6792$ & $x_{1}: 0.4405$ & $x_{1}: 0.7744$ \\
\hline & $x_{2}: 0.5806$ & $x_{2}: 0.6112$ & $x_{2}: 0.3559$ & $x_{2}: 0.6358$ \\
\hline & $x_{3}: 0.6034$ & $x_{3}: 0.6408$ & $x_{3}: 0.3975$ & $x_{3}: 0.6936$ \\
\hline & $x_{4}: 0.5742$ & $x_{4}: 0.6237$ & $x_{4}: 0.3604$ & $x_{4}: 0.6635$ \\
\hline & $x_{5}: 0.5484$ & $x_{5}: 0.5741$ & $x_{5}: 0.3008$ & $x_{5}: 0.5609$ \\
\hline & $x_{1} \succ x_{3} \succ x_{2} \succ$ & $x_{1} \succ x_{3} \succ x_{4} \succ$ & $x_{1} \succ x_{3} \succ x_{4} \succ$ & $x_{1} \succ x_{3} \succ x_{4} \succ$ \\
\hline & $x_{4}>x_{5}$ & $x_{2} \succ x_{5}$ & $x_{2}>x_{5}$ & $x_{2}>x_{5}$ \\
\hline \multirow{7}{*}{$P_{\alpha_{t}, B_{2},}\left(\bigotimes_{\boxminus=1}^{(\otimes A} \mathrm{A}\right)$} & $x_{1}: 0.3100$ & $x_{1}: 0.4133$ & $x_{1}: 0.3100$ & $x_{1}: 0.5402$ \\
\hline & $x_{2}: 0.2133$ & $x_{2}: 0.2967$ & $x_{2}: 0.2100$ & $x_{2}: 0.3252$ \\
\hline & $x_{3}: 0.2600$ & $x_{3}: 0.3367$ & $x_{3}: 0.2600$ & $x_{3}: 0.4019$ \\
\hline & $x_{4}: 0.2133$ & $x_{4}: 0.3433$ & $x_{4}: 0.2133$ & $x_{4}: 0.3901$ \\
\hline & $x_{5}: 0.1900$ & $x_{5}: 0.2533$ & $x_{5}: 0.1433$ & $x_{5}: 0.2390$ \\
\hline & $x_{1} \succ x_{3} \succ x_{2} \sim$ & $x_{1} \succ x_{4} \succ x_{3} \succ$ & $x_{1} \succ x_{3} \succ x_{4} \succ$ & $x_{1} \succ x_{3} \succ x_{4} \succ$ \\
\hline & $x_{4} \succ x_{5}$ & $x_{2}>x_{5}$ & $x_{2} \succ x_{5}$ & $x_{2}>x_{5}$ \\
\hline \multirow{6}{*}{ 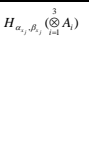 } & $x_{1}:-0.0830$ & $x_{1}:-0.0153$ & $x_{1}:-0.3090$ & $x_{1}: 0.0878$ \\
\hline & $\begin{array}{l}x_{2}:-0.1530 \\
x_{2}-0.1180\end{array}$ & $\begin{array}{l}x_{2}:-0.0687 \\
x_{2}-0.0374\end{array}$ & $\begin{array}{l}x_{2}:-0.3290 \\
x_{2}=-0.300\end{array}$ & $\begin{array}{l}x_{2}:-0.0488 \\
x=0.0134\end{array}$ \\
\hline & $x_{4}:-0.1507$ & $x_{4}:-0.0840$ & $X_{4}:-0.3540$ & $X_{4}:-0.0492$ \\
\hline & $x_{5}:-0.1997$ & $x_{5}:-0.1180$ & $x_{5}:-0.3630$ & $x_{5}:-0.1274$ \\
\hline & $x_{1} \succ x_{3} \succ x_{4} \succ$ & $x_{1} \succ x_{3} \succ x_{2} \succ$ & $x_{3} \succ x_{1} \succ x_{2} \succ$ & $x_{1} \succ x_{3} \succ x_{2} \succ$ \\
\hline & $x_{2}>x_{5}$ & $x_{4}>x_{5}$ & & $x_{4} \succ x_{5}$ \\
\hline \multirow{7}{*}{ 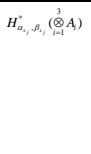 } & $x_{1}:-0.1724$ & $x_{1}:-0.0948$ & $x_{1}:-0.5871$ & $\begin{array}{ll}x_{1}: 0.0508 \\
\end{array}$ \\
\hline & $x_{2}:-0.2454$ & $x_{2}:-0.1508$ & $x_{2}:-0.6165$ & $x_{2}:-0.1196$ \\
\hline & $x_{3}:-0.2122$ & $x_{3}:-0.1184$ & $x_{3}:-0.6011$ & $x_{3}:-0.0452$ \\
\hline & $x_{4}:-0.2353$ & $x_{4}:-0.1592$ & $x_{4}:-0.6172$ & $x_{4}:-0.1079$ \\
\hline & $x_{5}:-0.2879$ & $x_{5}:-0.1964$ & $x_{5}:-0.6374$ & $x_{5}:-0.2115$ \\
\hline & $x_{1} \succ x_{3} \succ x_{4} \succ$ & $x_{1} \succ x_{3} \succ x_{2} \succ$ & $x_{1} \succ x_{3} \succ x_{2} \succ$ & $x_{1} \succ x_{3} \succ x_{4} \succ$ \\
\hline & $x_{2} \succ x_{5}$ & $x_{4} \succ x_{5}$ & $x_{4} \succ x_{5}$ & $x_{2} \succ x_{5}$ \\
\hline \multirow{7}{*}{$Q_{\alpha_{\alpha_{i}}, \beta_{i, j}}\left(\left(_{i=1}^{3} \mathrm{~A}\right)\right.$} & $x_{1}: 0.1000$ & $x_{1}: 0.0967$ & $x_{1}:-0.5000$ & $x_{1}: 0.2235$ \\
\hline & $x_{2}: 0.0967$ & $x_{2}: 0.1133$ & $x_{2}:-0.5000$ & $x_{2}: 0.1419$ \\
\hline & $x_{3}: 0.1000$ & $x_{3}: 0.1233$ & $x_{3}:-0.5000$ & $x_{3}: 0.1886$ \\
\hline & $x_{4}: 0.1000$ & $x_{4}: 0.0700$ & $x_{4}:-0.5000$ & $x_{4}: 0.1168$ \\
\hline & $x_{5}: 0.0533$ & $x_{5}: 0.0900$ & $x_{5}:-0.5000$ & $x_{5}: 0.0757$ \\
\hline & $x_{1} \sim x_{3} \sim x_{4} \succ$ & $x_{3} \succ x_{2} \succ x_{1} \succ$ & $x_{1} \sim x_{2} \sim x_{3} \sim$ & $x_{1} \succ x_{3} \succ x_{2} \succ$ \\
\hline & $x_{2} \succ x_{5}$ & $x_{5} \succ x_{4}$ & $x_{4} \sim x_{5}$ & $x_{4} \succ x_{5}$ \\
\hline
\end{tabular}
for all $x_{j} \in X$. The resulting Algorithms (I) and (II) analyses are summarized in Tables 1 and 2, respectively.

Tab. 1: The results of Algorithm (I) in the case of $\kappa_{1}=\kappa_{2}=1$ in the numerical example. 


\section{Conclusions}

In this study, we consider the influences of optimism and pessimism to develop different decision models and methods for multiple criteria decision making problems in an intuitionistic fuzzy environment. On the basis of the measurement tool estimations defined on IFSs, we utilize optimistic and pessimistic point operators to determine the influences of optimism and pessimism, respectively, and apply to multi-criteria decision analysis. For each point operator, we investigate and discuss several important properties to acquire a thoughtful understanding in concrete meanings of the relevant operations and relations. In addition, we develop an approach to deal with the effects caused by optimism and pessimism, consisting of changes in overall judgments and in separate evaluations of each alternative with respect to each criterion. The corresponding algorithms are provided to relate optimism and pessimism to multi-criteria decision analysis under the intuitionistic fuzzy decision setting.

Tab. 2: The results of Algorithm (II) in the case of $\kappa_{1}=\kappa_{2}=1$ in the numerical example.

\begin{tabular}{|c|c|c|c|c|}
\hline \multirow{3}{*}{$\begin{array}{l}\text { Suitability } \\
\text { function }\end{array}$} & \multicolumn{2}{|c|}{$\begin{array}{l}\text { Fixed point operator } \\
\end{array}$} & \multicolumn{2}{|c|}{ Variant point operator } \\
\hline & $\alpha_{x_{j}}=0.4$ & $\alpha_{x_{j}}=0.6$ & $\alpha_{x_{i}}=0.2$ & $\alpha_{x_{i}}=\mu_{i j} /\left(\mu_{i}+v_{i j}\right)$ \\
\hline & $\beta_{x_{i}}=0.3$ & $\beta_{x_{j}}=0.4$ & $\beta_{x_{j}}=0.7$ & $\beta_{x_{i}}=v_{i j} /\left(\mu_{i j}+v_{i_{i}}\right)$ \\
\hline \multirow{6}{*}{ 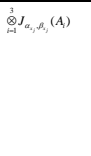 } & $x_{1}: 0.5673$ & $x_{1}: 0.6120$ & $x_{1}: 0.4293$ & $x_{1}: 0.6226$ \\
\hline & $\begin{array}{l}x_{2}: 0.4957 \\
x: 0.5297\end{array}$ & $x_{2}: 0.5020$ & $x_{2}: 0.3377$ & $x_{2}: 0.4765$ \\
\hline & $X_{4}: 0.5023$ & $\begin{array}{l}x_{3}: 0.5460 \\
x_{4}: 0.5313\end{array}$ & $\begin{array}{l}x_{3}: 0.3817 \\
x_{4}=0.3450\end{array}$ & $\begin{array}{l}x_{3}: 0.5537 \\
x .05326\end{array}$ \\
\hline & $x_{5}: 0 c .4513$ & $\begin{array}{l}x_{5}: 0.4493 \\
x_{1}\end{array}$ & $\begin{array}{l}x_{5}: 0.0 .2800 \\
x_{1}\end{array}$ & $\begin{array}{l}X: 0.0326 \\
X_{5}: 0.4375\end{array}$ \\
\hline & $x_{1} \succ x_{3} \succ x_{4} \succ$ & $x_{1} \succ x_{3} \succ x_{4} \succ$ & $x_{1} \succ x_{3} \succ x_{4} \succ$ & $x_{1} \succ x_{3} \succ x_{4} \succ$ \\
\hline & $x_{2}>x_{5}$ & $x_{2} \succ x_{5}$ & $x_{2} \succ x_{5}$ & \\
\hline \multirow{7}{*}{$\bigotimes_{-1}^{3} J_{\alpha_{i}, \beta_{i}}^{*},(A)$} & $x_{1:}: 0.6196$ & $\begin{array}{ll}1: 0.6792 \\
\end{array}$ & $x_{1}: 0.4405$ & $x_{1}: 0.7042$ \\
\hline & $\begin{array}{l}x_{2}: 0.5806 \\
x_{2}=0.6034\end{array}$ & $x_{2}: 0.6112$ & $x_{2}: 0.3559$ & $x_{2}: 0.5684$ \\
\hline & $x_{3}: 0.6034$ & $x_{3}: 0.6408$ & $x_{3}: 0.3975$ & $x_{3}: 0.6247$ \\
\hline & $x_{4}: 0.5742$ & $x_{4}: 0.6237$ & $x_{4}: 0.3604$ & $x_{4}: 0.6242$ \\
\hline & $x_{5}: 0.5484$ & $x_{5}: 0.5741$ & $x_{5}: 0.3008$ & $x_{5}: 0.5450$ \\
\hline & $x_{1} \succ x_{3} \succ x_{2} \succ$ & $x_{1} \succ x_{3} \succ x_{4} \succ$ & $x_{1} \succ x_{3} \succ x_{4} \succ$ & $x_{1} \succ x_{3} \succ x_{4} \succ$ \\
\hline & $x_{4}>x_{5}$ & $x_{2} \succ x_{5}$ & $x_{2} \succ x_{5}$ & $x_{2} \succ x_{5}$ \\
\hline \multirow{7}{*}{$\bigotimes_{i=1}^{3} P_{a_{x_{i}}, \beta_{i j}}\left(A_{i}\right)$} & $\begin{array}{ll}x_{1}: & 0.3567 \\
\end{array}$ & $x_{1}: 0.4900$ & $x_{1}: 0.3100$ & $\begin{array}{ll}x_{1}: 0.5018 \\
\end{array}$ \\
\hline & $x_{2}: 0.3367$ & $x_{2:}: 0.3900$ & $x_{2}: 0.2100$ & $x_{2}: 0.3234$ \\
\hline & $x_{3}: 0.3500$ & $x_{3}: 0.4000$ & $x_{3}: 0.2600$ & $x_{3}: 0.4221$ \\
\hline & $x_{4}: 0.2833$ & x4: 0.3567 & X4: 0.2133 & $x_{4}: 0.3853$ \\
\hline & $x_{5}: 0.2367$ & $x_{5}: 0.3067$ & $x_{5}: 0.1433$ & $x_{5}: 0.2510$ \\
\hline & $x_{1} \succ x_{3} \succ x_{2} \succ$ & $x_{1} \succ x_{3} \succ x_{2} \sim$ & $x_{1} \succ x_{3} \succ x_{4} \succ$ & $x_{1} \succ x_{3} \succ x_{4} \succ$ \\
\hline & $x_{4}>x_{5}$ & $x_{4}>x_{5}$ & $x_{2} \succ x_{5}$ & $x_{2} \succ x_{5}$ \\
\hline \multirow{7}{*}{ 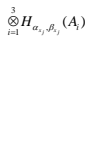 } & $x_{1}:-0.0830$ & $x_{1}:-0.0153$ & $x_{1}:-0.3090$ & $x_{1}: 0.0643$ \\
\hline & $x_{2}:-0.1530$ & $x_{2}:-0.0687$ & $x_{2}:-0.3290$ & $x_{2}:-0.0131$ \\
\hline & $x_{3}:-0.1180$ & $x_{3}:-0.0347$ & $x_{3}:-0.3080$ & $x_{3}: 0.0771$ \\
\hline & $x_{4}:-0.1507$ & $x_{4}:-0.0840$ & $x_{4}:-0.3540$ & $x_{4}:-0.0354$ \\
\hline & $x_{5}:-0.1997$ & $x_{5}:-0.1180$ & $x_{5}:-0.3630$ & $x_{5}:-0.0988$ \\
\hline & $x_{1} \succ x_{3} \succ x_{4} \succ$ & $x_{1} \succ x_{3} \succ x_{2} \succ$ & $x_{3} \succ x_{1} \succ x_{2} \succ$ & $x_{3} \succ x_{1} \succ x_{2} \succ$ \\
\hline & $x_{2}>x_{5}$ & $x_{4} \succ x_{5}$ & $x_{4} \succ x_{5}$ & $x_{4}>x_{5}$ \\
\hline
\end{tabular}

\begin{tabular}{|c|c|c|c|c|}
\hline \multirow[t]{2}{*}{$\otimes_{i=1}^{3} H_{\alpha_{i}, B_{4}}^{*}\left(A_{i}\right)$} & $\begin{array}{l}x_{1}:-0.1724 \\
x_{2}:-0.2454 \\
x_{3}:-0.2122 \\
x_{4}:-0.2353 \\
x_{5}:-0.2879\end{array}$ & $\begin{array}{l}x_{1}:-0.1345 \\
x_{2}:-0.1919 \\
x_{3}:-0.1603 \\
x_{4}:-0.1968 \\
x_{5}:-0.2356\end{array}$ & $\begin{array}{l}x_{1}:-0.5176 \\
x_{2}:-0.5446 \\
x_{3}:-0.5278 \\
x_{4}:-0.5514 \\
x_{5}:-0.5688\end{array}$ & $\begin{array}{l}x_{1}: 0.0251 \\
x_{2}:-0.0745 \\
x_{3}: 0.0164 \\
x_{4}:-0.0912 \\
x_{5}:-0.1778\end{array}$ \\
\hline & $\begin{array}{l}x_{1} \succ x_{3} \succ x_{4} \succ \\
x_{2} \succ x_{5}\end{array}$ & $\begin{array}{l}x_{1} \succ x_{3} \succ x_{2} \succ \\
x_{4} \succ x_{5}\end{array}$ & $\begin{array}{l}x_{1} \succ x_{3} \succ x_{2} \succ \\
x_{4} \succ x_{5}\end{array}$ & $\begin{array}{l}x_{1}>x_{3} \succ x_{2} \succ \\
x_{4} \succ x_{5}\end{array}$ \\
\hline$\bigotimes_{i=1}^{3} Q_{a_{t,}, B_{i, j}}(A)$ & $\begin{array}{l}x_{1}: 0.0533 \\
x_{2}:-0.0267 \\
x_{3}: 0.0100 \\
x_{4}: 0.0300 \\
x_{5}: 0.0067\end{array}$ & $\begin{array}{l}x_{1}: 0.0200 \\
x_{2}: 0.0200 \\
x_{3}: 0.0600 \\
x_{4}: 0.0067 \\
x_{5}: 0.0367\end{array}$ & $\begin{array}{l}x_{1}:-0.5000 \\
x_{2}:-0.5000 \\
x_{3}:-0.5000 \\
x_{4}:-0.5000 \\
x_{5}:-0.5000\end{array}$ & $\begin{array}{l}x_{1}: 0.1851 \\
x_{2}: 0.1400 \\
x_{3}: 0.2087 \\
x_{4}: 0.1120 \\
x_{5}: 0.0876\end{array}$ \\
\hline & $\begin{array}{l}x_{1} \succ x_{4} \succ x_{3} \succ \\
x_{5} \succ x_{2}\end{array}$ & $\begin{array}{l}x_{3} \succ x_{4} \succ x_{5} \succ \\
x_{1} \sim x_{2}\end{array}$ & $\begin{array}{l}x_{1} \sim x_{2} \sim x_{3} \sim \\
x_{4} \sim x_{5}\end{array}$ & $\begin{array}{l}x_{3} \succ x_{x} \succ x_{2} \succ \\
x_{1} \succ x_{5}\end{array}$ \\
\hline
\end{tabular}

\section{References}

[1] K.T. Atanassov, "Intuitionistic Fuzzy Sets," Fuzzy Sets and Systems, vol. 20, pp. 87-96, 1986.

[2] K.T. Atanassov, Intuitionistic Fuzzy Sets: Theory and Applications, New York: Physica-Verlag, Heidelberg, 1999.

[3] K. T. Atanassov, G. Pasi, and R. Yager, "Intuitionistic Fuzzy Interpretations of Multi-criteria Multi-person and Multi-measurement Tool Decision Making," International Journal of Systems Science, vol. 36, no. 14, pp. 859-868, 2005.

[4] D. Dubois, S. Gottwald, P. Hajek, J. Kacprzyk, and H. Prade, "Terminological Difficulties in Fuzzy Set Theory-The Case of "Intuitionistic Fuzzy Sets”," Fuzzy Sets and Systems, vol. 156, pp. 485-491, 2005.

[5] L. Friedman, A. Weinberg, J. Webb, H. Cooper, and S. Bruce, "Skin Cancer Prevention and Early Detection Intentions and Behavior," American Journal of Preventive Medicine, vol. 11, pp. 59-65, 1995.

[6] D.-F. Li, "Multiattribute Decision Making Models and Methods Using Intuitionistic Fuzzy Sets,” Journal of Computer and System Sciences, vol. 70, no. 1, pp. 73-85, 2005.

[7] L. Lin, X.-H. Yuan, and Z.-Q. Xia, "Multicriteria Fuzzy Decisionmaking Methods Based on Intuitionistic Fuzzy Sets," Journal of Computer and Systems Sciences, vol. 73, no. 1, pp. 84-88, 2007. 
[8] M. Puri, and D.T. Robinson, "Optimism and Economic Choice," Journal of Financial Economics, vol. 86, pp. 71-99, 2007.

[9] L.J. Sanna, and E.C. Chang, "The Past is not What It Used to be: Optimists' Use of Retroactive Pessimism to Diminish the Setting of Failure," Journal of Research in Personality, vol. 37, pp. 388-404, 2003.

[10]Z. Xu, and R.R. Yager, "Dynamic Intuitionistic Fuzzy Mutli-attribute Decision Making," International Journal of Approximate Reasoning, vol. 48, no. 1, pp. 246-262, 2008.

Proceedings of the 11th Joint Conference on Information Sciences (2008)

Published by Atlantis Press (c) the authors 